\title{
Proceedings
}

Juntendo Medical Journal

2018. 64 (Suppl 1), 145

\section{Localization of Damage in Rat Quadriceps Femoris Muscle Induced by Downhill Running}

\author{
TOMOKO FUNAKOSHI*1) 2), KeIgo OHTA*1), SHUICHI MACHIDA*1) \\ *1) Graduate School of Health and Sports Science, Juntendo University, Chiba, Japan, \\ *2) Molecular Regulation of Aging, Tokyo Metropolitan Institute of Gerontlogy, Tokyo, Japan
}

\begin{abstract}
Introduction: Injury can be induced in myofibers by strenuous and unaccustomed exercise or exercise comprising lengthening. Eccentric muscle contraction, such as downhill running, accounts for more significant injury to limb muscles. The well-characterized sequence of events during muscle repair includes an infiltration of inflammatory cells to the injury site followed by the activation of satellite cells to repair and replenish the injured tissue. To understand whether damage induced by downhill running would be localized to restricted area or not, we investigated damaged region with accumulation of mononuclear cells in the quadriceps femoris muscles of rat after downhill running histologically combined with monitoring levels of creatine kinase in blood.
\end{abstract}

Materials and Methods: We divided nine 15-16-week-old Sprague-Dawley male rats (body weight, $454 \pm 14 \mathrm{~g}$ ) into control (unexercised) and exercise groups and performed downhill running. Animals in the exercise group were made to run on a treadmill ( $-16^{\circ}$ incline) at $16 \mathrm{~m} / \mathrm{min}$ for $90 \mathrm{~min}$ : 5 -min bouts (18 bouts) with 2 -min rests. After downhill running, we obtained blood samples from the tails of rats after $0,24,48,72$, and $92 \mathrm{~h}$ and evaluate CK levels with Reflotron blood analyzer. To assess the basal level, we collected blood samples before exercise. The quadriceps femoris muscles were removed from the exercise group on days 3, 5, and 8 after the completion of downhill running and were frozen in isopentane cooled with liquid nitrogen. We cut serial cross-sections $(10 \mu \mathrm{m}$ thickness) of mid-belly muscles using a cryostat at $-20^{\circ} \mathrm{C}$ for the histological evaluation of the muscle damage induced by downhill running. We examined these sections after staining with hematoxylin and eosin. Furthermore, we immunostained sections with anti-Pax7 antibody after fixation in $4 \%$ formaldehyde and visualized with a diaminobenzidine reaction to identify muscle progenitor satellite cells. The nuclei were counterstained with hematoxylin.

Results: CK activity was elevated after downhill running, with two peaks at 0 and $48 \mathrm{~h}$ after exercise; however, the level decreased at $24 \mathrm{~h}$. After the second peak at $48 \mathrm{~h}$, CK activity declined again; the level remained unaltered in unexercised rats at any sampling time. In line with the results of previous studies, our results suggest that downhill protocol induces muscle damage. HE-stained cross-sections of the quadriceps femoris muscles from exercised rats demonstrated damage at $3 \mathrm{~d}$ after running. We observed accumulations of mononuclear cells in the interstitium and within some muscle fibers. Furthermore, the damage was more significant and widespread at $5 \mathrm{~d}$. Muscle damage was non-uniform and notable damage was predominantly observed in a restricted area within the vastus intermedius. The number of Pax7-positive satellite cells increased within the damaged areas. At $8 \mathrm{~d}$ after running, the damaged area and number of mononuclear cells decreased, thereby indicating lesion repair.

Conclusion: This study suggests that downhill running induces rat muscle damage in a restricted area within the vastus intermedius.

Key words: downhill running, muscle damage, creatine kinase

\footnotetext{
Corresponding author: Shuichi Machida

Graduate School of Health and Sports Science, Juntendo University

1-1 Hirakagakuendai, Inzai-shi, Chiba 270-1695, Japan

TEL: +81-476-98-1001 FAX: +81-476-98-1011 E-mail: machidas@juntendo.ac.jp

〔Received Nov. 28, 2017〕〔Accepted Feb. 13, 2018〕
}

Copyright (C) 2018 The Juntendo Medical Society. This is an open access article distributed under the terms of Creative Commons Attribution License (CC BY), which permits unrestricted use, distribution, and reproduction in any medium, provided the original source is properly credited. doi: 10.14789/jmj.2018.64.JMJ18-P511 